\title{
Observation of dark and bright pulses in Q-switched erbium doped fiber laser using graphene nano-platelets as saturable absorber
}

\author{
Nur Hidayah Muhamad Apandi ${ }^{1}$, Siti Nur Fatin Zuikafly ${ }^{2}$, Nabilah Kasim ${ }^{3}$, Mohd Ambri Mohamed ${ }^{4}$, \\ Sulaiman Wadi Harun ${ }^{5}$, Fauzan Ahmad \\ ${ }^{1,2,6}$ Malaysia-Japan International Institute of Technology, Universiti Teknologi Malaysia, Jalan Sultan Yahya Petra, \\ 54100 Kuala Lumpur, Malaysia \\ ${ }^{3}$ Department of Physics, Faculty of Science, Universiti Teknologi Malaysia, 81310 Johor Bahru, Malaysia \\ ${ }^{4}$ Institute of Microengineering and Nanoelectronics, Universiti Kebangsaan Malaysia, 43600 Bangi, Selangor, Malaysia \\ ${ }^{5}$ Department of Electrical Engineering, University of Malaya, 50603 Kuala Lumpur, Malaysia
}

\begin{tabular}{|c|c|}
\hline Article Info & ABSTRACT \\
\hline Article history: & \multirow{10}{*}{$\begin{array}{l}\text { In this paper, a passively Q-switched Erbium doped fiber laser (EDFL) by } \\
\text { residing Graphene nanoplatelets (GnPs) embedded in polyvinyl alcohol } \\
\text { (PVA) based saturable absorber (SA) is demonstrated. To aid the dispersion } \\
\text { of GNPs, a surfactant is used and then it is mixed with polyvinyl alcohol } \\
\text { (PVA) as host polymer to develop GnPs-PVA film based passive SA. The } \\
\text { GnPs-PVA based film then integrated in laser cavity in ring cavity } \\
\text { configuration for pulse laser generation. The experimental works show that } \\
\text { the proposed passive SA operates at input pump power range from } 77 \mathrm{~mW} \text { to } \\
128 \mathrm{~mW} \text { with a tunable repetition rate from } 78.4 \mathrm{kHz} \text { to } 114.8 \mathrm{kHz} \text { and a } \\
\text { shortest pulse width of } 3.69 \mu \text { s. The laser produces maximum instantaneous } \\
\text { output peak power and pulse energy of } 7.3 \mathrm{~mW} \text { and } 30.46 \mathrm{~nJ} \text {, respectively } \\
\text { and accompanied by signal to noise ratio (SNR) of } 64 \mathrm{~dB} \text {. } \\
\text { Copyright } \odot 2019 \text { Institute of Advanced Engineering and Science. } \\
\text { All rights reserved. }\end{array}$} \\
\hline Received Mar 29, 2019 & \\
\hline Revised Jun 10, 2019 & \\
\hline Accepted Jul 1, 2019 & \\
\hline Keywords: & \\
\hline Dark and bright pulse & \\
\hline Fiber laser & \\
\hline Graphene nano platelets & \\
\hline Q-switched & \\
\hline Saturable absorber & \\
\hline
\end{tabular}

Corresponding Author:

Fauzan Ahmad,

Malaysia-Japan International Institute of Technology,

University Teknologi Malaysia,

Jalan Sultan Yahya Petra, 54100 Kuala Lumpur, Malaysia.

Email: fauzan.kl@utm.my

\section{INTRODUCTION}

The first fiber laser was successfully demonstrated in 1960s [1] and it has intensively enhanced the development for both telecommunication [2] and sensor technology [3]. There are two well-known approach to generate pulse fiber laser either active or passive Q-switching. Passive technique become favorable as it offers compactness, low cost, flexibility and simple design compared to active technique. Several methods have been proposed to achieve passive Q-switched fiber laser like nonlinear polarization (NPR) and real saturable absorber (SA) [4]. However, introducing SA into the fiber laser cavity is considered as a decisive way and provide simplicity. Fundamentally, pulsed laser can operate in two different regime which are bright regime and dark regime [5]. Dark pulses are normally referred to a train of intensity dips in a continuous wave background of the laser emission however, most of the pulsed laser operates in bright regime. Graphene based SAs have shown outstanding potential due to its intrinsic characteristic which is ultrafast recovery time and ultra-broadband operation. The intraband relaxation time around 10-150 fs [6] create an opportunity to produce shorter pulse than other materials used as SA. An ultra-broadband operation enable a broad working wavelength which enable us to use different gain media such as Ytterbium, Erbium and Thulium doped fiber.

Graphene nanaplatelets $(\mathrm{GnP})$ is one of the graphene derivatives which $\mathrm{GnP}$ is stacks with 3-10 layer of graphene [7], while graphene with number of layer more than 10 is characterize as graphite. The 
quality of $\mathrm{GnP}$ is alike graphene due to the similarity configuration of pristine basal plane with an enhance of optical, electrical and mechanical properties as compared to graphene oxide (GO) [7]. Basically there are four mainly approaches to synthesize graphene and its derivatives. One is through mechanical exfoliation [8] where scotch tape is used to peel off a layer or layers of graphite from bulk. This method is simple and gives better electronic properties of the samples but have low yield. The number of layers of which the graphite is peeled off is also random and cannot be controlled. There is also a method using insulating surfaces for epitaxial growth [9]. The third was CVD on metals such as $\mathrm{Ni}$ [10] and $\mathrm{Cu}$ [11] and epitaxial growth on certain facet of metal such as ruthenium (0001) [12]. This method is favourable due to its ability to produce a large area of uniform graphene film [13]. For Q-switched pulsed laser, graphene CVD approach has been demonstrated by placing a graphene based thin film between two fiber connectors [14]. They reported to produces repetition rate of $34.72 \mathrm{kHz}$ to $53.2 \mathrm{kHz}$ with a shortest pulse width of $3.2 \mu \mathrm{s}$ within input pump power of $57.2 \mathrm{~mW}$ to $74.23 \mathrm{~mW}$. Last but not least, there is also a fabrication method using liquid phase exfoliation (LPE) that can produce graphene from graphite and its derivatives and compounds [15]. The LPE method to fabricate graphene polymer composite has been demonstrated by Popa et al [16] where they able to produce a Q-switched pulse laser with a repetition rate of $36 \mathrm{kHz}$ to $103 \mathrm{kHz}$, a shortest pulse width of 2 $\mu \mathrm{s}$ and signal-to-noise ratio (SNR) of $42 \mathrm{~dB}$. A wider range of repetition rates were also demonstrated [17] ranging from $1.39 \mathrm{kHz}$ to $206.61 \mathrm{kHz}$ using a Graphene based SA fabricated through optical deposition of graphene-N-methyl Pyrrolidone (NMP). Cao et al [18] produced the SA by optical deposition of graphene-Dimethylformamide (DMF) suspension and able to generate Q-switched pulse laser with repetition rate of $8.50 \mathrm{kHz}$ to $29.05 \mathrm{kHz}$ and a shortest pulse width of $4.6 \mu \mathrm{s}$.

\section{RESEARCH METHOD}

The Graphene nano-platelets (GnP) powder obtained from Chengdu Organic Chemicals Co. Ltd., with purity, number of layers, thickness, diameter and density of $>99.5 \mathrm{wt} \%,<20,4-20 \mathrm{~nm}, 5-10 \mu \mathrm{m}$ and $0.6 \mathrm{~g} / \mathrm{cm} 3$, respectively, were used without any purification. In order to develop Graphene nano-platelets based passive saturable absorber, two separate suspension was prepared. The first one is to prepare dispersed graphene nano-platelets and the other one is polyvinyl alcohol (PVA) which act as binder, before both were mixed together. The GnP nano powder is weighed to about $40 \mathrm{mg}$ and added to $40 \mathrm{ml}$ of sodium dodecyl sulphate (SDS) in $1 \%$ deionized (DI) water.The mixture was ultrasonicated using a tip sonicator with pulse cycle of $0.5 \mathrm{~s}$ at $90 \mathrm{~W} / \mathrm{cm}^{2}$ for 3 hours. After that, the suspension was centrifuge at $1000 \mathrm{rpm}$ for one hour and $70 \%$ of the upper suspension was decanted leaving undispersed GNP at the bottom side. Then, the host polymer which is polyvinyl alcohol (PVA) $\left(\mathrm{Mw}=89 \times 10^{3} \mathrm{~g} / \mathrm{Mol}\right)$ (Sigma Aldrich) was prepared by dissolving it into $120 \mathrm{ml}$ of DI water. The GnP-PVA composite was then develop by mixing the dispersed GnP suspension and the PVA solution with one to one ratio. Through a one hour ultrasonic bath process, a homogenous GnP-PVA composite was obtained before it was poured onto a petri dish and let dry at room temperature. After 48 hours, a GnP-PVA film formed and can be peeled off thepetri dish. The film's thickness is approximately $30 \mu \mathrm{m}$. The FESEM image and Raman spectroscopy investigation of the SA is shown in Figure 1 and Figure 2, respectively.

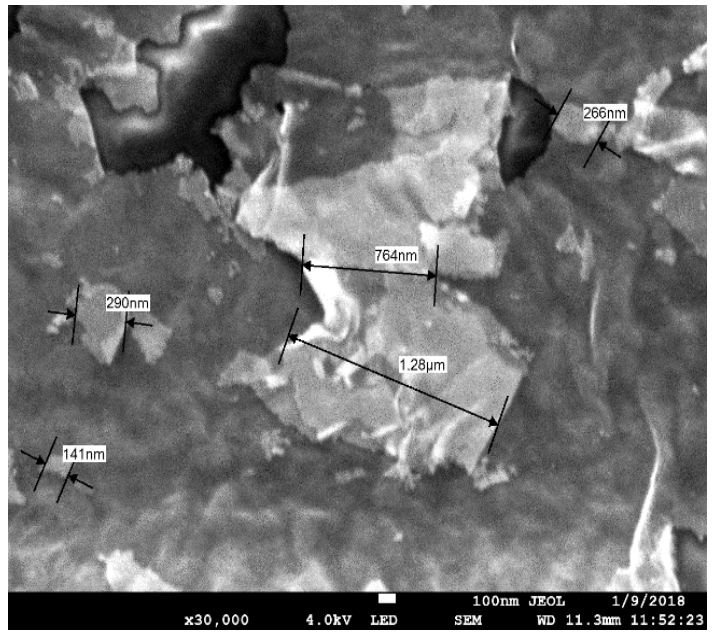

Figure 1. FESEM image of GnP-PVA film

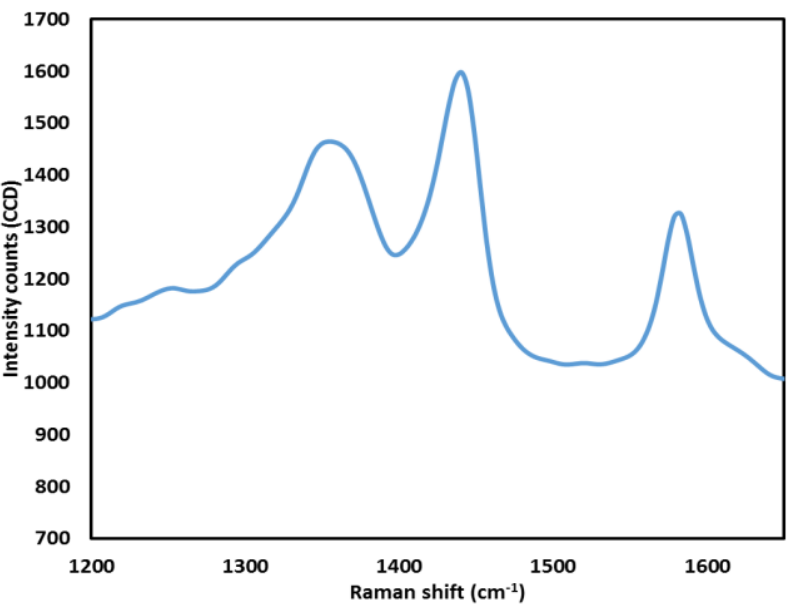

Figure 2. Raman spectroscopy of GnP-PVA film 
The experimental setup is as shown in Figure 3. To integrate the GnP-PVA film as the SA into the cavity, the film is cut into a small piece and attached to one end of a fiber ferrule with the aid of index matching gel. A $2 \mathrm{~m}$ long Erbium doped fiber is used as the gain medium, along with a 980/1550 $\mathrm{nm}$ wavelength division multiplexer (WDM), an isolator to ensure uni-directional propagation of the oscillating light, and a $90 / 10$ output coupler to tap out $10 \%$ of the light for output characterization. A $3 \mathrm{~dB}$ coupler is used to allow for simultaneous observation of the pulse produced through an optical spectrum analyser (OSA)/Optical power meter (OPM) and oscilloscope (OSC)/Radio frequency spectrum analyser (RFSA).

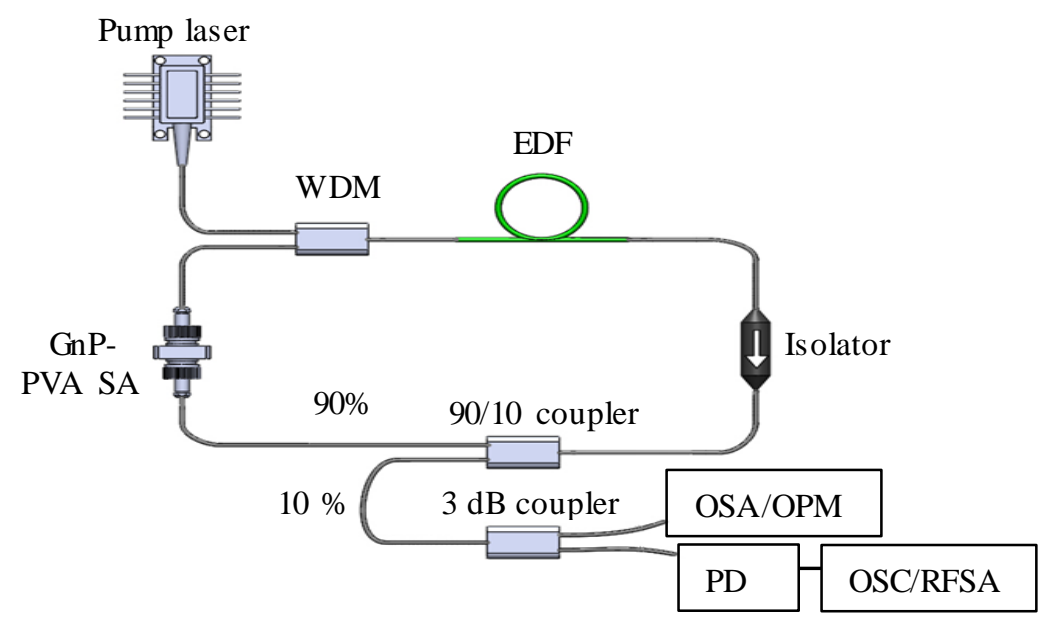

Figure 3. Experimental set-up of fiber laser in ring cavity

\section{RESULTS AND ANALYSIS}

A $3 \mathrm{~dB}$ spectral broadening of around $1 \mathrm{~nm}$ occurred as the wavelength shifted from $1571.67 \mathrm{~nm}$ during CW lasing to $1562.30 \mathrm{~nm}$ due to the insertion of the GnP-PVA film SA, as shown in Figure 4. The self-started dark pulsed Q-switched fiber laser occurs at input pump power of $77 \mathrm{~mW}$ and continue to input pump power of $90 \mathrm{~mW}$ as in Figure 5. Above $90 \mathrm{~mW}$, the Q-switched pulse oscillate at bright pulse or ordinary Q-switched pulse until the maximum pump power of $128 \mathrm{~mW}$.

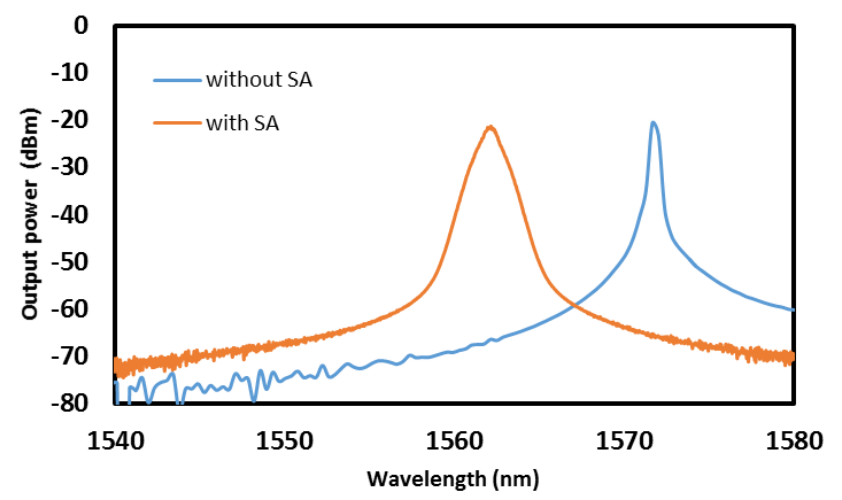

Figure 4. OSA trace without SA and with SA 


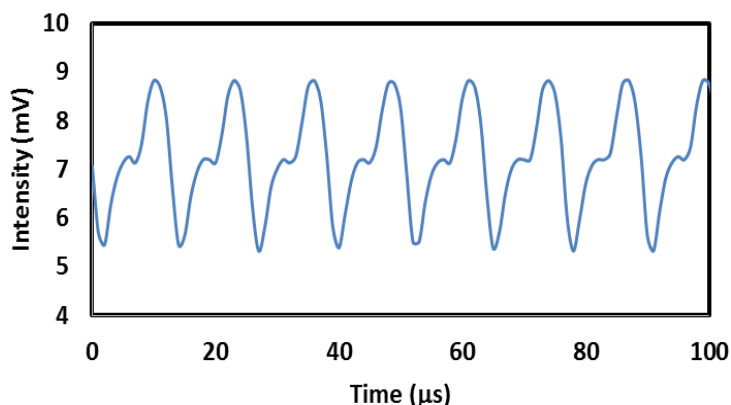

(a)

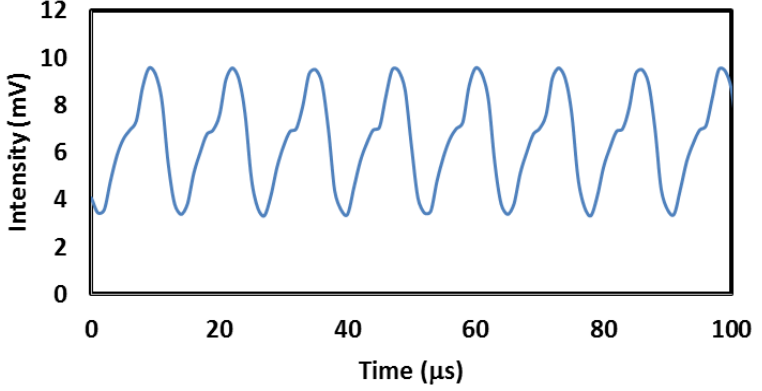

(b)

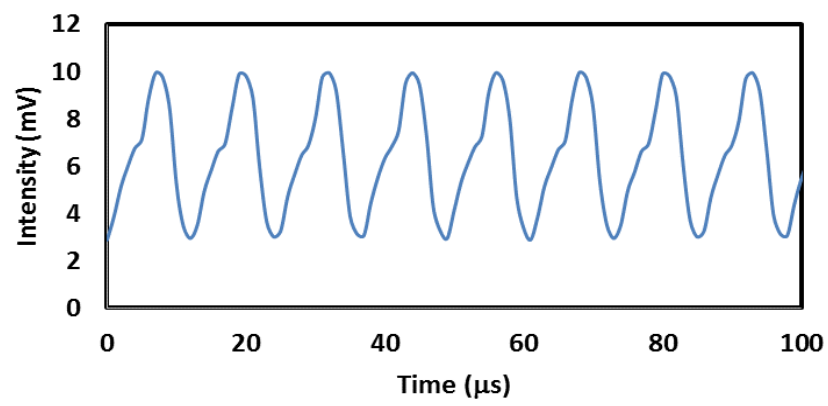

(c)

Figure 5. Dark pulse emission of the proposed EDFL at different pump power, (a) $77 \mathrm{~mW}$, (b) $84 \mathrm{~mW}$, (c) $90 \mathrm{~mW}$

The oscilloscope trace at maximum input pump power of $128 \mathrm{~mW}$ is shown in Figure 6 with the inset showing the shortest pulse width of $3.69 \mu$ s. The repetition rate were tunable from $78.4 \mathrm{kHz}$ to 114.8 $\mathrm{kHz}$ with increasing pump power. These value provide a highest value if compared to previous works $[16,18-24]$. The relationship between the pump power and the repetition rate as well as the pulse width is portrayed in Figure 7. As the pump power is increased gradually, the repetition rate increases as well from $78.4 \mathrm{kHz}$ to $114.8 \mathrm{kHz}$ while the pulse width is reduced from $8.12 \mu \mathrm{s}$ to $3.69 \mu \mathrm{s}$. This observation corresponds to the trending of pulse width and repetition rate that commonly observed in passively Q-switched lasers [25]. The shortest pulse width generated in this experiment is slightly better than previous work in [18-20, 25]. From the measured pulse repetition rate, pulse width and average output power, the pulse energy and peak power are calculated and being tabulated in Figure 8. As shown, both peak power and pulse energy increased as the input pump power increased. The maximum pulse energy and instantaneous peak power produced by the generated pulse is $30.46 \mathrm{~nJ}$ and $7.3 \mathrm{~mW}$, respectively. Beyond $115 \mathrm{~mW}$ input pump power, the pulse energy is toward decreasing trends due to the slight difference in average output power as pulse energy, $E=\mathrm{P}_{\mathrm{av}} / \mathrm{f}$. The generated instantaneous peak power is better than in [22] and the pulse energy higher than in $[19,22,24]$. The measured signal to noise ratio is around $64 \mathrm{~dB}$ as shown in Figure 9, at maximum input pump power, shows the high stability of the generated pulse and higher than previous works in $[18,26]$. 


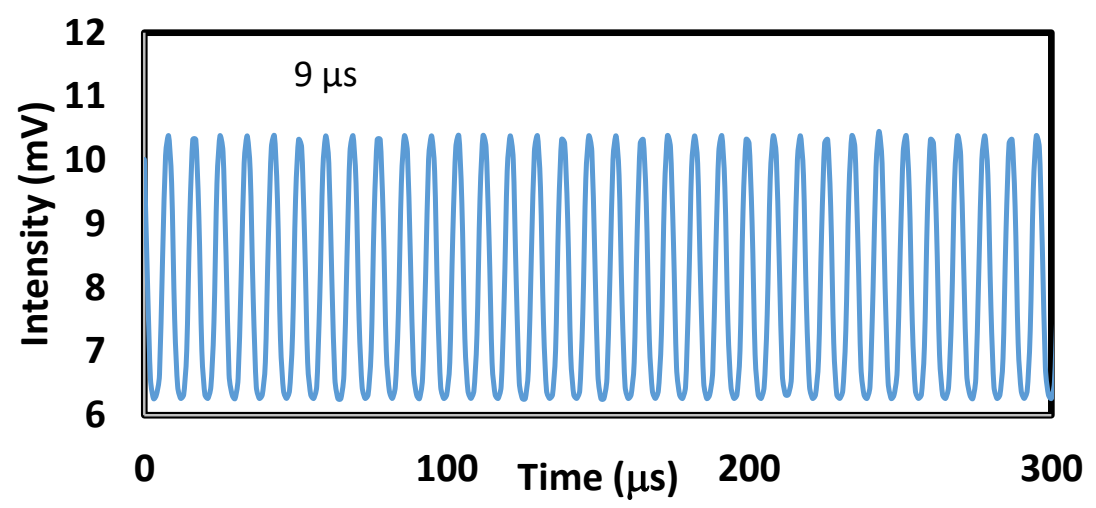

Figure 6. Pulse train of bright regime at $128 \mathrm{~mW}$; inset: Shortest pulse width of $3.69 \mu \mathrm{s}$

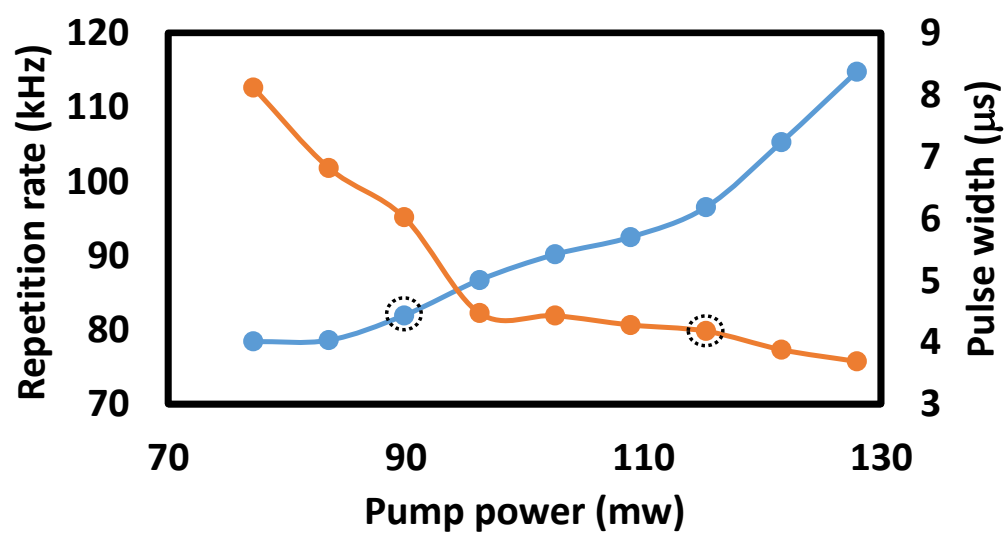

Figure 7. Repetiton rate and pulse width as a function of pump power

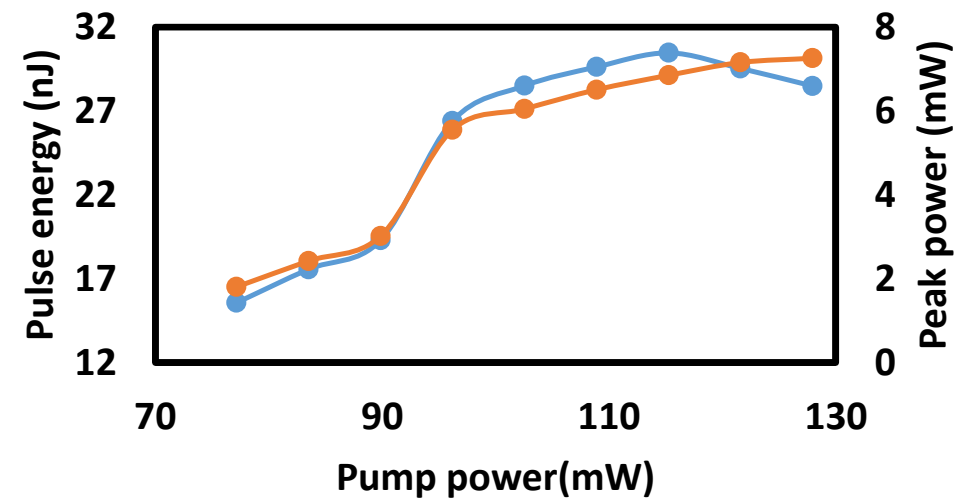

Figure 8. Peak power and pulse energy as a function of pump power 


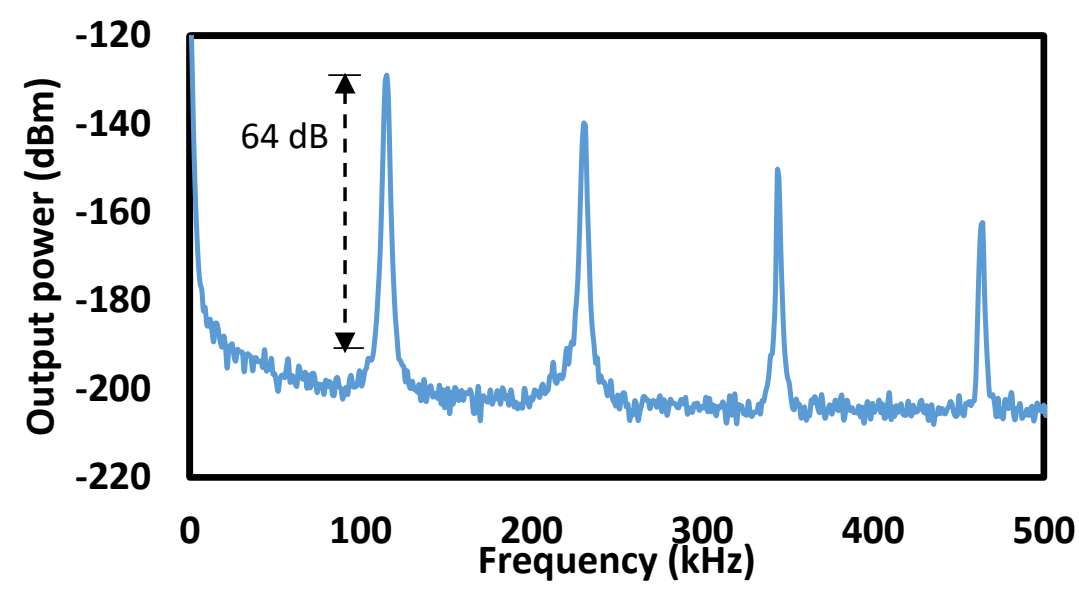

Figure 9. RFSA measurement of first beat note at a repetition rate of $114.8 \mathrm{kHz}$

\section{CONCLUSION}

Graphene nanoplatelets-PVA film based passive Q-switched EDFL was successfully demonstrated. This approach to fabricate GnP-PVA is both scalable, affording the possibility of high-volume production, and versatile in terms of being well suited to chemical functionalization. These advantages mean that graphene and its derivatives produced by the colloidal suspension method are good nanofillers to incorporate into the polymer to form functional composites.

\section{ACKNOWLEDGEMENTS}

The authors acknowledge Universiti Teknologi Malaysia (UTM) for supporting this research work under UTM R\&D Fund grant no: 4J304, UTM grant (R.J130000.7826.4F928) and Malaysia Japan International Institute of Technology (MJIT) Scholarship Fund.

\section{REFERENCES}

[1] N. H. Apandi, F. Ahmad, S. Ambran, M. Yamada, S.W. Harun, "Bismuth (III) Telluride $\left(\mathrm{Bi}_{2} \mathrm{Te}_{3}\right) \mathrm{Based}$ Topological Insulator Embedded in PVA as Passive Saturable Absorber in Erbium-Doped Fiber Laser," In IOP Conference Series: Materials Science and Engineering, vol. 210(1), pp. 012032, 2017.

[2] K. Bhatnagar. "Latest trends in fiber optics communication," International research Journal of Engineering and technology, vol. 3(11), pp. 45-54, 2016.

[3] W. G. Xie, Y. N. Zhang, P. Z. Wang, and J. Z. Wang. "Optical fiber sensors based on fiber ring laser demodulation technology," Sensors, vol. 18, pp. 3-25, 2018.

[4] M. Wu, Y. Chen, H. Zhang and S. Wen, "Nanosecond Q-switched Erbium-Doped Fiber Laser With Wide Pulse-Repetition-Rate Range Based on Topological Insulator," in IEEE Journal of Quantum Electronics, vol. 50, no. 6, pp. 393-396, June 2014.

[5] H. Pan, A. Zhang, and Z. Tong. "Generation of dark and bright pulses in an soa-based Q-switched fiber laser," Laser Physics, vol. 27, pp. 085006, 2017.

[6] P. A. George, J. Strait, J. Dawlaty, S. Shivaraman, M. Chandrashekhar, F. Rana, M.G. Spencer, "Ultrafast optical-pump terahertz-probe spectroscopy of the carrier relaxation and recombination dynamics in epitaxial graphene," Nano letters., vol. 8(12), pp. 4248-5,2008.

[7] S. N. Kazi, A. Badarudin, M. N. Zubir, H. N. Ming, Misran M. Misran, E. Sadeghinezhad, M. Mehrali, N. I. Syuhada, "Investigation on the use of graphene oxide as novel surfactant to stabilize weakly charged graphene nanoplatelets," Nanoscale research letters, vol. 10(1), pp. 212, 2015.

[8] M. Yi and Z. Shen. " A review on mechanical exfoliation for the scalable production of graphene," Journal of Materials Chemistry A, vol. 22, 2015.

[9] J. Wang, Z. Xiong, J. Yu, H. Yn, X. Wang, L. Peng, Y. Wang, X. Wang, T. Jiang, L. Cao, W. Wu, C. Wang, and L. Zhang. "Epitaxial growth of graphene thin film by pulsed laser deposition," Micro \& Nano Letters, vol. 10(11), pp. 649-652, 2015.

[10] K. L. Ding, B. Xiu, C. Xu, J. Huang, and D. Guo. "Preparation and saturable absorption property of graphene on the optic fiber side by transferring CVD-Graphene grown on Ni," IOP Conf. Series: Materials Science and Engineering, vol. 230, pp. 012029, 2017. 
[11] G. Sobon, J. Sotor, I. pasternak, A. Krajewska, W. Strupinski, K. A. Abramski. "Thulium-doped all-fiber laser mode-locked by CVD-graphene/PMMA saturable absorber," Optics Express, vol. 21(10), pp. 12797-12802, 2013.

[12] P. W. Sutter, J. I. Flege, E. A. Sutter, "Epitaxial graphene on ruthenium." Nature materials, vol. 7(5), pp. 406, 2008.

[13] G. R. Yazdi, T. Iakimov, and R. Yakimova. "Epitaxial graphene on SiC: A review of groeth and characterization," Crystals, vol. 6, pp. 1-45, 2016.

[14] L. Q. Zhang, Z. Zhuo, J. X. Wang, Y. Z. Wang, "Passively Q-switched fiber laser based on graphene saturable absorber." Laser Physics, vol. 22(2), pp. 433-6, 2012.

[15] Z. Sun, T. Hasan, A.C. Ferrari, "Ultrafast lasers mode-locked by nanotubes and graphene." Physica E: Low-dimensional Systems and Nanostructures, vol. 44(6), pp. 1082-91, 2012.

[16] D. Popa, Z. Sun, T. Hasan, F. Torrisi, F. Wang, A. C. Ferrari, "Graphene Q-switched, tunable fiber laser." Applied Physics Letters, vol. 98(7), pp. 073106, 2011.

[17] H. Ahmad, M. Z. Zulkifli, F. D. Muhammad, A. Z. Zulkifli, and S. W. Harun, "Tunable Graphene-based Q-switched erbium-doped fiber laser using fiber Bragg grating," Journal of Modern Optics, vol. 60(3), pp. 202-212, 2013.

[18] W. J. Cao, H. Y. Wang, A. P. Luo, Z. C. Luo and W. C. Xu, "Graphene based, $50 \mathrm{~nm}$ wide-band tunable passively Q-switched fiber laser," Laser Physics Letters, vol. 9(1), pp. 54-58, 2012.

[19] Y. K. Yap, N. M. Huang, S. W. Harun, H. Ahmad, "Graphene Oxide-Based Q-switched Erbium-Doped Fiber Laser," Chin. Phys. Lett., vol. 30(2), pp. 024208, 2013.

[20] L. He-Ping et al., "A compact graphene Q-switched erbium-doped fiber laser using optical circulator and tunable fiber Bragg grating." Chinese Physics B.,vol. 23(2), pp. 024209, 2013.

[21] B. Lu , H. Chen , M. Jiang, X. Chen, Z. Ren, J. Bai, "Graphene-based passive Q-switching for a $2 \mu \mathrm{m}$ thulium-doped fiber laser." Laser Physics., vol. 23(4), pp. 045111, 2013.

[22] Z. Q. Luo, M. Zhou, J. Weng, G. M. Huang, H. Y. Xu, C. C. Ye, and Z. P. Cai, "Graphene-based passively Q-switched dual-wavelength erbium-doped fiber laser," Opt. Lett., vol. 35, pp. 3709, 2010.

[23] H. Chu et al., "Dual-Wavelength Passively Q-switched Nd, Mg: $\mathrm{LiTaO}_{3}$ Laser With a Monolayer Graphene as Saturable Absorber." IEEE Journal of Selected Topics in Quantum Electronics, vol. 21(1), pp. 343-7, 2015.

[24] Z. T. Wang, Y. Chen, C. J. Zhao, H. Zhang and S. C. Wen, "Switchable Dual-Wavelength Synchronously Q-switched Erbium-Doped Fiber Laser Based on Graphene Saturable Absorber," in IEEE Photonics Journal, vol. 4, no. 3, pp. 869-876, June 2012.

[25] X. C. Lin et al., "Multi-walled carbon nanotube as a saturable absorber for a passively mode-locked Nd: YVO4 laser, Laser Physics Letters, vol. 10(5), pp. 055805, 2013.

[26] F. D. Muhammad, M. Z. Zulkifli and H. Ahmad, "Graphene based Q-switched tunable S-band fiber laser incorporating arrayed waveguide gratings (AWG)," Journal of Nonlinear Optical Physics \& Materials, vol. 23(1), pp. 1450004, 2014.

\section{BIOGRAPHIES OF AUTHORS}
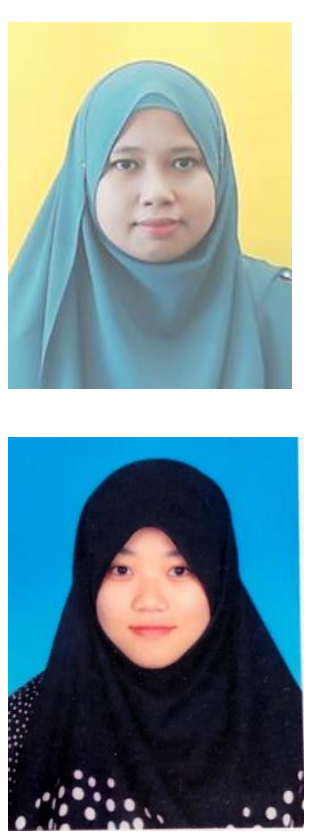

Nur Hidayah Muhamad Apandi received Bachelor degree from University Malaysia Perlis (2015) and Master degree (2018) in Electronic System Engineering from Universiti Teknologi Malaysia, Malaysia. She is currently pursuing her Ph.D in photonics at the Malaysia-Japan International Institute of Technology (MJJIT), Universiti Teknologi Malaysia. She is a member of Optical System and Devices (ODESYS) Ikohza of MJIIT and her research interest includes 1D material application for pulsed laser generation.

Siti Nur Fatin Zuikafly received her Bachelor degree (2016) and Master degree (2018) in Electronic System Engineering from Universiti Teknologi Malaysia, Malaysia. She is currently pursuing her Ph.D in photonics at the Malaysia-Japan International Institute of Technology (MJJIT), Universiti Teknologi Malaysia. She is a member of Optical System and Devices (ODESYS) Ikohza of MJIIT and her research interest includes 2D material application for pulsed laser generation. 

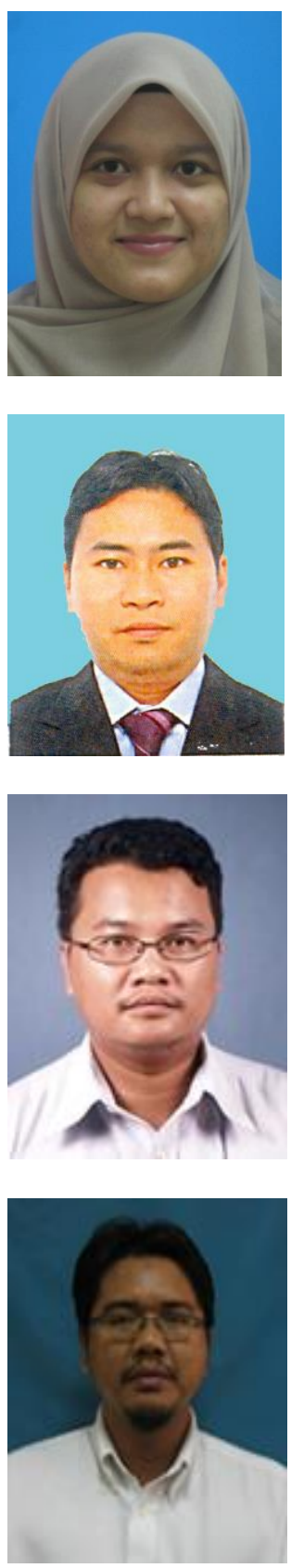

Nabilah Kasim is a senior lecturer in Department of Physics, Faculty of Science, Universiti Teknologi Malaysia. She has involved in fiber optic experiments utilizing graphene and carbon nanotubes as saturable absorber since 2014 and still active in the study of pulsed laser. The current research interest revolve in generating pulsed laser by using various rare earth elements as the gain medium and using different saturable absorber.

Mohd Ambri Mohamed received B.E. degree in Materials Engineering from Tokyo University of Science, Japan in 2004 and M. Sc. And Ph. D. in Materials Science from Japan Advanced Institute of Science and Technology, Japan in 2007 and 2010. He is currently an Associate Professor at Institute of Microengineering and Nanoelectronics, Universiti Kebangsaan Malaysia. His current research interest includes carbon derivatives and 2D related materials application in nanoelectronics devices and epitaxial growth of compound semiconductors.

Sulaiman Wadi Harun received the B.E. degree in electrical and electronics system engineering from the Nagaoka University of Technology, Nagaoka, Japan, in 1996, and the M.Sc. and Ph.D. degrees in photonics from the University of Malaya, Kuala Lumpur, Malaysia, in 2001 and 2004, respectively. He is currently a Full Professor with the Faculty of Engineering, University of Malaya. His current research interests include fiber optic active and passive devices.

Fauzan Ahmad received the Bachelor's in Mechatronics from Universiti Teknologi Malaysia in 1999, Master's Degree (Image processing) and PhD Degree in Electrical Engineering (Photonics) from University of Malaya in 2007 and 2014, respectively. He is currently a senior lecturer at Department of Electronic Systems Engineering, Malaysia-Japan International Institute of Technology (MJIIT), Universiti Teknologi Malaysia. He is a member of Optical System and Devices (ODESYS) Ikohza of MJIIT and his research interest includes nano material application for pulsed laser generation and optical fiber sensor. 